\title{
Multiwavelength $\mathrm{OH}$ observations of Mon R2 during 4765 $\mathrm{MHz} \mathrm{OH}$ maser flaring
}

\author{
Derck P. Smits \\ Department of Mathematics, Applied Mathematics $\&$ Astronomy, \\ University of South Africa, PO Box 392, UNISA, 0003, South Africa
}

\begin{abstract}
During the spectacular $4765 \mathrm{MHz}$ excited $\mathrm{OH}$ maser flaring episodes observed in Mon R2 from 1994 to 1998 (Smits et al. 1998), observations were made at the line frequencies of other $\mathrm{OH}$ transitions. No excited $\mathrm{OH}$ lines were detected, nor were any 1612 or $1720 \mathrm{MHz}$ $\mathrm{OH}$ masers found in Mon R2. 1665 and $1667 \mathrm{MHz} \mathrm{OH}$ masers were present and underwent small changes. With a peak flux of $\sim 75 \mathrm{Jy}$, the null detections and small variations at other wavelengths put strong constraints on models of $\mathrm{OH}$ masers.
\end{abstract}

\section{Introduction}

Maser emission from rotationally excited levels of $\mathrm{OH}$ has only been found in star-forming regions. The most common excited $\mathrm{OH}$ masers are the $6035 \mathrm{MHz}$ line from the first excited rotational level and the $4765 \mathrm{MHz}$ line from the lowest level of the ${ }^{2} \Pi_{1 / 2}$ chain. A theoretical treatment of radiative transfer applied to a multilevel OH system by Field \& Gray (1988) showed that the growth and saturation of any one maser line is intimately linked to the behaviour of other $\mathrm{OH}$ maser lines. Observations have indicated that the $4765 \mathrm{MHz}$ masers might be associated with $1720 \mathrm{MHz}$ ground-state $\mathrm{OH}$ masers and $6035 \mathrm{MHz}$ masers. MacLeod (1997) listed the numbers of 1720,6035 and $4765 \mathrm{MHz}$ masers detected towards those sources which had been observed at all three frequencies. Eight of the eleven $4765 \mathrm{MHz}$ maser sources in his sample also had detections at 1720 and $6035 \mathrm{MHz}$ at some stage (not necessarily simultaneously). This suggests that the three different maser species could occur in regions with similar physical conditions, or they could change from one type to another as the region evolves. The $4765 \mathrm{MHz}$ excited $\mathrm{OH}$ maser flaring reported by Smits, Cohen \& Hutawarakorn (1998) in Mon R2 provided an opportunity to test correlations between the lines of $\mathrm{OH}$ from different rotational levels.

Single dish monitoring and a MERLIN observation of Mon R2 showed that the $4765 \mathrm{MHz}$ emission consisted of multiple maser spots located in the region known as IRS3. The spots had about $15 \%$ linear polarization aligned at a position angle of $14^{\circ}$. With a peak flux density of $\sim 75 \mathrm{Jy}$ the Mon R2 4765 $\mathrm{MHz}$ maser emission was twenty times stronger than any other known $4765 \mathrm{MHz}$ source, and hence sets strong limits on the strengths of other $\mathrm{OH}$ maser lines. 


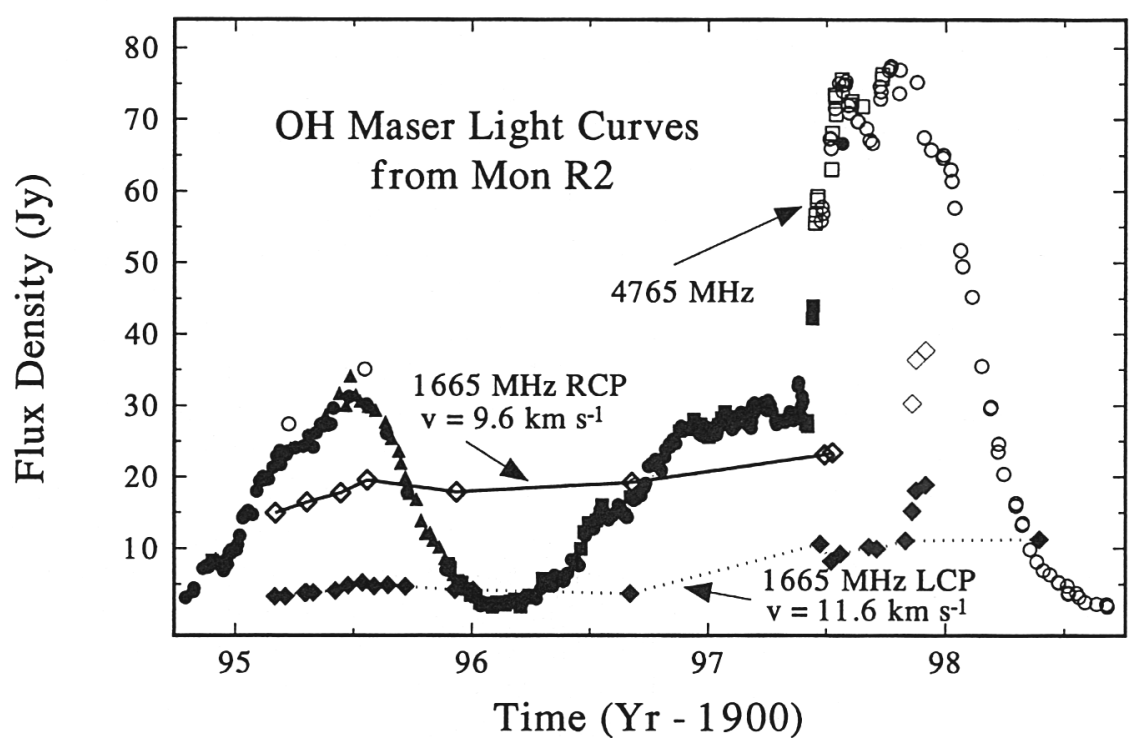

Figure 1. Temporal variations of the peak flux density at $4765 \mathrm{MHz}$ and of one peak each in the $1665 \mathrm{MHz}$ LCP and RCP spectra.

\section{Observations}

Observations using the $26 \mathrm{~m}$ radio telescope of the Hartebeesthoek Radio Astronomy Observatory (HartRAO) were made at frequencies of the four $18 \mathrm{~cm}$ groundstate transitions, the four $6 \mathrm{GHz}$ transitions from the first excited rotational level and the three $4.7 \mathrm{GHz}$ transitions of the lowest level in the ${ }^{2} \Pi_{1 / 2}$ chain of $\mathrm{OH}$. One observation was made at $8.189 \mathrm{GHz}$, the frequency of the $F=3 \Rightarrow F=3$ transition of the ${ }^{2} \Pi_{1 / 2} J=5 / 2$ level.

The complete light-curve for the single, broad line of $4765 \mathrm{MHz}$ excited $\mathrm{OH}$ maser emission from Mon R2 is shown in Figure 1. The last observation shown in this figure was made on 03 September 1998, by which time the flux density had dropped below 2 Jy. When observations commenced again on 06 December 1998 there was no detectable signal (to a $3 \sigma$ limit of at least $0.5 \mathrm{Jy}$ ). Monitoring of Mon R2 has continued approximately every two weeks since then, but no sign of any further activity had been found up to February 2001.

With the exception of the $4765 \mathrm{MHz}$ emission, no other $\mathrm{OH}$ maser activity was found from excited levels of $\mathrm{OH}$ to a limit of $\sim 0.5-1 \mathrm{Jy}$ (i.e. to about $1 \%$ of the $4765 \mathrm{MHz}$ flux density). Observations at 4660 and $4750 \mathrm{MHz}$ were made about every two months from late 1994 to September 1996, and once in August 1997 when the $4765 \mathrm{MHz}$ flux was about $75 \mathrm{Jy}$. The $6 \mathrm{GHz}$ lines were looked for 
once early in 1995 at both circular polarizations, and on three occasions when the $4765 \mathrm{MHz}$ flux was above $60 \mathrm{Jy}$. At no stage were any of the $6 \mathrm{GHz} \mathrm{OH}$ lines detected above the RMS noise. In November 1996 ( $\mathrm{Yr}=96.87) 9 \mathrm{hrs}$ of data were obtained at $8189.587 \mathrm{MHz}$ towards Mon R2. Nothing was found to a level of $0.3 \mathrm{Jy}$.

Ground-state $\mathrm{OH} 1612 \mathrm{MHz}$ emission consists of two broad peaks with fluxes of 1.0 and $1.2 \mathrm{Jy}$ at velocities of 9.1 and $11.5 \mathrm{~km} \mathrm{~s}^{-1}$ respectively extending over a velocity range from 6.5 to $12.5 \mathrm{~km} \mathrm{~s}^{-1}$. This emission appears to have remained constant since 1973 when it was first detected by Downes et al. (1975). A similar pattern occurs at $1720 \mathrm{MHz}$, but in absorption rather than in emission. Such conjugate behaviour of the $\mathrm{OH}$ satellite lines is common in star-forming regions and is due to quasi-thermal emission from the molecular cloud surrounding the region. $1720 \mathrm{MHz}$ observations have been made since 1995 up to February 2001, but no maser signatures have been seen in the spectra at any stage.

Absorption troughs are also present over the same velocity range in the 1665 and $1667 \mathrm{MHz}$ spectra, but the shape of the absorption features are obscured by maser emission sitting in the absorption trough. At $1667 \mathrm{MHz}$ the dominant maser lines occur at velocities of $9.8 \mathrm{~km} \mathrm{~s}^{-1}$ in RCP and $10.6 \mathrm{~km} \mathrm{~s}^{-1}$ at LCP. The emission from these two features has a high degree of circular polarization. These lines are broad enough to indicate that they consist of multiple, unresolved spots of emission. From February 1995 to February 1996 the $1667 \mathrm{MHz}$ masers underwent no noticeable changes. Two observations made in mid-1997 showed that the RCP component had increased from its 1995 value of $1.7 \mathrm{Jy}$ to about $3.5 \mathrm{Jy}$. The flux of the LCP component ( 1 Jy) did not change.
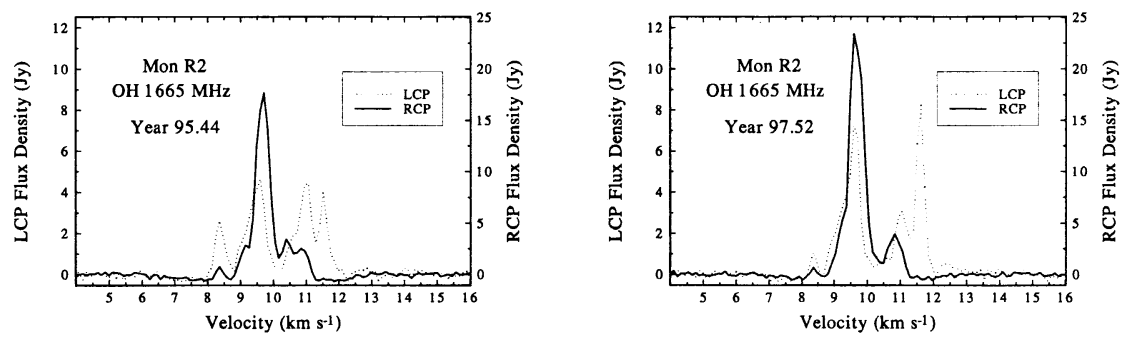

Figure 2. $1665 \mathrm{MHz}$ LCP (dotted line) and RCP (solid line) spectra of Mon R2 taken 2 years apart. The scale for LCP is on the left of the plot, and that for RCP on the right.

The $1665 \mathrm{MHz}$ maser emission in Mon R2 has been known to vary since its discovery by Downes et al. (1975). LCP and RCP spectra taken in June 1995 and August 1997 are shown in Figure 2 (note that the LCP and RCP fluxes use different scales). Variations of the spectra at the two epochs are clearly visible. The lines were broad and the peaks shifted channels during the observation period. This suggest that the lines are blends of more than one maser component which grow and die out at different times. 
A plot of the temporal variations of the flux density in the RCP channel at $9.6 \mathrm{~km} \mathrm{~s}^{-1}$ and the LCP channel corresponding to $11.6 \mathrm{~km} \mathrm{~s}^{-1}$ are shown in Figure 1 superimposed on the $4765 \mathrm{MHz}$ light curve. The points connected by lines (dotted and solid) are from HartRAO, the other three points are from Nançay radiotelescope. The observations were made on 10 and 17 November, and 01 December, and show that the $1665 \mathrm{MHz}$ fluxes of these two peaks underwent an impulsive increase at this time.

A second epoch of $4.7 \mathrm{GHz}$ MERLIN observations with full polarization was obtained in December 1997 when the $4765 \mathrm{MHz}$ flux density was $\sim 60 \mathrm{Jy}$. The maps revealed that most of the emission came from a new maser spot which also had about $15 \%$ linear polarization at a position angle of $14^{\circ}$. Observations at 4660 and $4750 \mathrm{MHz}$ showed no maser emission to a level of $30 \mathrm{mJy}$, which is 2000 times weaker than the $4765 \mathrm{MHz}$ flux density.

\section{Discussion}

Multiline pumping models of $\mathrm{OH}$ masers do not account for the behaviour of the $\mathrm{OH}$ masers observed in Mon $\mathrm{R} 2$ during the large $4765 \mathrm{MHz}$ excited $\mathrm{OH}$ flaring episodes from 1994 to 1998 . No other excited $\mathrm{OH}$ masers were found in this region throughout the flaring episodes, nor was there any sign of 1720 $\mathrm{MHz}$ groundstate $\mathrm{OH}$ maser activity. Groundstate 1665 and $1667 \mathrm{MHz} \mathrm{OH}$ masers have been present in this source since at least 1973 (Downes et al. 1975). Although variable, the changes were generally slower and smaller than those seen in the $4765 \mathrm{MHz}$ spectra. On occassions, the $4765 \mathrm{MHz}$ intensity was more than four times stronger than the $1665 \mathrm{MHz}$ groundstate emission. The impulsive increase seen in the $1665 \mathrm{MHz}$ Nançay radiotelescope data occurred about 5.5 months after that at $4765 \mathrm{MHz}$, indicating clearly that the 1665 and $4765 \mathrm{MHz}$ lines are formed in distinctly different regions. The $\mathrm{OH}$ masers in Mon R2 do not appear to have any connection with each other, and therefore must be pumped independently.

All spots of $4765 \mathrm{MHz}$ maser emission had linear polarization of $15 \%$ lying at a position angle of $14^{\circ}$. This is the only $4765 \mathrm{MHz}$ maser known to have any polarization. Knapp \& Brown (1976) reported a high degree of linear polarization in at least two of the $1665 \mathrm{MHz}$ maser lines. Full polarization $1665 \mathrm{MHz}$ MERLIN observations of Mon R2, made on 7 May 1998, are being processed.

\section{References}

Downes, D., Winnberg, A., Goss, W. M., \& Johansson, L. E. B. 1975, A\&A, 44, 243

Field, D., \& Gray, M. D. 1988, MNRAS, 234, 353

Knapp, G. R., \& Brown, R. L. 1976, ApJ, 204, 21

MacLeod, G. C. 1997, MNRAS, 285, 635

Smits, D. P., Cohen, R. J., \& Hutawarakorn, B. 1998, MNRAS, 296, L11 